\title{
A UNIVERSAL METRIC FOR THE CANONICAL BUNDLE OF A HOLOMORPHIC FAMILY OF PROJECTIVE ALGEBRAIC MANIFOLDS*
}

\author{
DROR VAROLIN ${ }^{\dagger}$
}

Dedicated to M. Salah Baouendi on the occasion of his 70th birthday

Key words. Deformation Invariance of Plurigenera, Ohsawa-Takegoshi $L^{2}$-extension

AMS subject classifications. $32 \mathrm{~L} 1014 \mathrm{~F} 10$

1. Introduction. In his celebrated work [S-98, S-02], Siu proved that the plurigenera of any algebraic manifold are invariant in families. More precisely, let $\pi: \mathscr{X} \rightarrow \mathbb{D}$ be a holomorphic submersion (i.e., $d \pi$ is nowhere zero) from a complex manifold $\mathscr{X}$ to the unit disk $\mathbb{D}$, and assume that every fiber $\mathscr{X}_{t}:=\pi^{-1}(t)$ is a compact projective manifold. Then for every $m \in \mathbb{N}$, the function $P_{m}: \mathbb{D} \rightarrow \mathbb{N}$ defined by $P_{m}(t):=h^{0}\left(\mathscr{X}_{t}, m K_{\mathscr{X}_{t}}\right)$ is constant.

Siu's approach to the problem begins with the observation that the function $P_{m}$ is upper semi-continuous. Thus in order to prove that $P_{m}$ is continuous (hence constant) it suffices to show that given a global holomorphic section $s$ of $m K \mathscr{X}_{0}$, there is a family of global holomorphic sections $s_{t}$ of $\mathscr{X}_{t}$, for all $t$ in a neighborhood of 0 , that varies holomorphically with $t$ and satisfies $s_{0}=s$.

To prove such an extension theorem, Siu establishes a generalization of the Ohsawa-Takegoshi Extension Theorem to the setting of complex submanifolds of a Kahler manifold having codimension 1 and cut out by a single, bounded holomorphic function. This theorem, which we will discuss below, requires the existence of a singular Hermitian metric on the ambient manifold having non-negative curvature current, with respect to which the section to be extended is $L^{2}$. Thus in the presence of the extension theorem, the approach reduces to construction of such a metric.

The case where the fibers $\mathscr{X}_{t}$ of our holomorphic family are of general type was treated in [S-98]. In this setting, Siu produced a single singular Hermitian metric $e^{-\kappa}$ for $K_{X}$ so that every $m$-canonical section is $L^{2}$ with respect to $e^{-(m-1) \kappa}$.

However, in the case where the fibers $\mathscr{X}_{t}$ of our holomorphic family are assumed only to be algebraic, and not necessarily of general type, Siu's proof in [S-02] does not construct a single metric as in the case of general type. Instead, Siu constructs for every section $s$ of $m K_{\mathscr{X}_{0}}$ a singular Hermitian metric for $m K_{\mathscr{X}}$ of non-negative curvature so that $s$ is $L^{2}$ with respect to this metric.

Definition. Let $\mathscr{X} \rightarrow \Delta$ be a holomorphic family of complex manifolds and $\mathscr{X}_{0}$ the cental fiber of $\mathscr{X}$. A universal canonical metric for the pair $\left(\mathscr{X}, \mathscr{X}_{0}\right)$ is a singular Hermitian metric $e^{-\kappa}$ for the canonical bundle $K_{\mathscr{X}}$ of $\mathscr{X}$ such that for every global holomorphic section $s \in H^{0}\left(\mathscr{X}_{0}, m K \mathscr{X}_{0}\right)$,

$$
\int_{\mathscr{X}_{0}}|s|^{2} e^{-(m-1) \kappa}<+\infty .
$$

\footnotetext{
*Received July 14, 2006; accepted for publication March 19, 2007.

${ }^{\dagger}$ Department of Mathematics, Stony Brook University, Stony Brook, NY 11794, USA (dror@math.sunysb.edu). Partially supported by an NSF grant.
} 
The goal of this paper is to prove that for any holomorphic family $\mathscr{X} \rightarrow \Delta$ of compact complex algebraic manifolds with central fiber $\mathscr{X}_{0}$, the pair $\left(\mathscr{X}, \mathscr{X}_{0}\right)$ has a universal canonical metric having non-negative curvature current. To this end, our main theorem is the following result.

TheOrem 1. Let $X$ be a complex manifold admitting a positive line bundle $A \rightarrow$ $X$, and $Z \subset X$ a smooth compact complex submanifold of codimension 1. Assume there is a subvariety $V \subset X$ not containing $Z$ such that $X-V$ is a Stein manifold. Let $T \in H^{0}(X, Z)$ be a holomorphic section of the line bundle associated to $Z$, thought of as a divisor. Let $E \rightarrow X$ be a holomorphic line bundle and denote by $K_{X}$ the canonical bundle of $X$. Assume we are given singular metrics $e^{-\varphi_{E}}$ for $E$ and $e^{-\varphi_{Z}}$ for the line bundle associated to $Z$.

Suppose in addition that the above data satisfy the following assumptions.

(R) The metrics $e^{-\varphi_{E}}$ and $e^{-\varphi_{Z}}$ restrict to singular metrics on $Z$.

(B)

$$
\sup _{X}|T|^{2} e^{-\varphi_{Z}}<+\infty .
$$

(G) For each $m>0$, the line bundles $p\left(K_{X}+Z+E\right)+A, 0 \leq p \leq m-1$, are globally generated, in the sense that a finite number of sections of $H^{0}\left(X, p\left(K_{X}+Z+\right.\right.$ $E)+A)$ generate the sheaf $\mathcal{O}_{X}\left(p\left(K_{X}+Z+E\right)+A\right)$.

(P) $\sqrt{-1} \partial \bar{\partial} \varphi_{E} \geq 0$ and there exists a constant $\mu$ such that $\mu \sqrt{-1} \partial \bar{\partial} \varphi_{E} \geq$ $\sqrt{-1} \partial \bar{\partial} \varphi_{Z}$

(T) The singular metric $e^{-\left(\varphi_{Z}+\varphi_{E}\right)} \mid Z$ has trivial multiplier ideal:

$$
\mathscr{I}\left(Z, e^{-\left(\varphi_{Z}+\varphi_{E}\right)} \mid Z\right)=\mathcal{O}_{Z} .
$$

Then there is a metric $e^{-\kappa}$ for $K_{X}+Z+E$ with the following properties:

(C) $\sqrt{-1} \partial \bar{\partial} \kappa \geq 0$.

(L) For every $m>0$ and every section $s \in H^{0}\left(Z, m\left(K_{Z}+E \mid Z\right)\right)$, $|s|^{2} e^{-\left((m-1) \kappa+\varphi_{E}+\varphi_{Z}\right)}$ is locally integrable.

(I) For every integer $m>0$ and every section $s \in H^{0}\left(Z, m\left(K_{Z}+E\right)\right)$,

$$
\int_{Z}|s|^{2} e^{-(m-1) \kappa+\varphi_{E}}<+\infty
$$

\section{REMARKS.}

(i) For the ambient manifold $X$, we have in mind the following two examples: either $X$ is compact complex projective (in which case the variety $V$ could be taken to be a hyperplane section of some embedding of $X$ ) or else $X$ is a family of compact complex algebraic manifolds. In the former case, it is well-known [S-98] that the hypothesis (G) holds for any sufficiently ample $A$, while in the latter case, one might have to shrink $X$ a little to obtain $(\mathrm{G})$. Of course, there are many other examples of such $X$.

(ii) Note that in condition (L), the local functions $|s|^{2} e^{-\left((m-1) \kappa+\varphi_{E}+\varphi_{Z}\right)}$ depend on the local trivializations of the line bundles in question. However, the local integrability condition is independent of these choices.

Together with a variant of the Ohsawa-Takegoshi Theorem (Theorem 4 below), Theorem 1 implies a generalization of Siu's extension theorem to the case where the normal bundle of the submanifold $Z$ is not necessarily trivial. The first extension 
theorem of this type was established by Takayama [Ta-05, Theorem 4.1] under some additional hypotheses. The general case was done in [V-06], where Theorem 4 was also established. (In the case where $Z$ is a fiber in a smooth familty, the result in [V-06] was also proved by Claudon in [C-06].) The argument here is related to that of [V-06], but the focus is on construction of the metric rather than on the extension theorem.

As a result of Theorem 1, we have the following corollary, which is our stated goal.

COROLlaRY 2. For every holomorphic family $\mathscr{X} \rightarrow \Delta$ of smooth projective varieties with central fiber $\mathscr{X}_{0}$, the pair $\left(\mathscr{X}, \mathscr{X}_{0}\right)$ has, perhaps after slightly shrinking the family, a universal canonical metric having non-negative curvature current.

Proof. Let $X$ be a family of compact projective manifolds $\pi: \mathscr{X} \rightarrow \mathbb{D}$, and $Z=\mathscr{X}_{0}$ the central fiber. Take $T=\pi, E=\mathcal{O}_{\mathscr{X}}$ and $\varphi_{E} \equiv 0$. Since $\mathscr{X}_{0}$ is cut out by a single holomorphic function, the line bundle associated to $\mathscr{X}_{0}$ is trivial. Take $\varphi_{Z} \equiv 0$. Then the hypotheses of Theorem 1 are satisfied, perhaps after shrinking the family, and we obtain a metric $e^{-\kappa}$ for $K_{\mathscr{X}}$ such that $\sqrt{-1} \partial \bar{\partial} \kappa \geq 0$ and $|s|^{2} e^{-(m-1) \kappa}$ is integrable for every integer $m>0$ and every section $s \in H^{0}\left(\mathscr{X}_{0}, m K_{\mathscr{X}_{0}}\right)$.

REMARK. Note that in the setting of families, the constant $\mu$ is not needed, and the hypotheses (L) and (I) are the same.

REMARK. In his paper [Ts-02], Tsuji has claimed the existence of a metric with the properties stated in Corollary 2. As in our approach, Tsuji's proof makes use of an infinite process. It seems that convergence of Tsuji's process was not checked; in fact, it is demonstrated in [S-02] that Tsuji's process, as well as any reasonable modification of it, diverges.

Proposition 3. For each integer $m>0$, fix a basis $s_{1}^{(m)}, \ldots, s_{N_{m}}^{(m)}$ of $H^{0}\left(X, m\left(K_{Z}+E \mid Z\right)\right)$. Choose constants $\varepsilon_{m}$ such that the metric

$$
\kappa_{0}:=\log \left(\sum_{m=1}^{\infty} \varepsilon_{m}\left(\sum_{\ell=1}^{N_{m}}\left|s_{\ell}^{(m)}\right|^{2}\right)^{1 / m}\right)
$$

is convergent. Suppose $e^{-\varphi_{E}}$ is locally integrable. Then for each $m>0$ and every $s \in H^{0}\left(X, m\left(K_{Z}+E \mid Z\right)\right)$,

$$
\int_{Z}|s|^{2} e^{-\left((m-1) \kappa_{0}+\varphi_{E}\right)}<+\infty
$$

Proof. Fix $s \in H^{0}\left(X, m\left(K_{Z}+E \mid Z\right)\right)$, and let $\kappa_{0, m}=\log \left(\sum_{\ell=1}^{N_{m}}\left|s_{\ell}^{(m)}\right|^{2}\right)^{1 / m}$. 
Note that $e^{-\kappa_{0}} \lesssim e^{-\kappa_{0, m}}$, and thus we have

$$
\begin{aligned}
& \int_{Z}|s|^{2} e^{-(m-1) \kappa_{0}+\varphi_{E}} \\
\lesssim & \int_{Z}|s|^{2} e^{-(m-1) \kappa_{0, m}+\varphi_{E}} \\
= & \int_{Z}|s|^{2 / m}\left(\frac{|s|^{2}}{\left|s_{1}^{(m)}\right|^{2}+\ldots+\left|s_{N_{m}}^{(m)}\right|^{2}}\right)^{(m-1) / m} e^{\gamma_{E}-\varphi_{E}} e^{-\gamma_{E}} \\
\lesssim & \int_{Z}|s|^{2 / m} e^{\gamma_{E}-\varphi_{E}} e^{-\gamma_{E}} \\
\lesssim & \left(\int_{Z}|s|^{2} e^{\gamma_{E}-\varphi_{E}} e^{-m \gamma_{E}} \omega^{-(n-1)(m-1)}\right)^{1 / m}\left(\int_{Z} e^{\gamma_{E}-\varphi_{E}} \omega^{n-1}\right)^{(m-1) / m},
\end{aligned}
$$

where $\omega$ is a fixed Kähler form for $Z$ and $e^{-\gamma_{Z}}$ is a smooth metric for $E \mid Z$. The last inequality is a consequence of Hölder's Inequality. Since $e^{-\varphi_{E}}$ is locally integrable, we are done.

A calculation similar to the proof of Proposition 3 shows that $|s|^{2} e^{-\left((m-1) \kappa_{0}+\varphi_{Z}+\varphi_{E}\right)}$ is locally integrable on $Z$. Thus in view of Proposition 3 , Theorem 1 follows if we construct a metric $e^{-\kappa}$ with non-negative curvature current such that $e^{-\kappa} \mid Z=e^{-\kappa_{0}}$. This is precisely what we do. We employ a technical simplification, due to Paun [P-05], of Siu's original idea of extending metrics using an Ohsawa-Takegoshi-type extension theorem for sections. Paun's simplification allows one to get rid of a rather difficult part of Siu's original proof; the use (and proof) of an effective version of global generation of multiplier ideal sheaves. As a consequence of Paun's methods, the present paper is also substantially shortened.

2. The Ohsawa-Takegoshi Extension theorem. Let $Y$ be a Kähler manifold of complex dimension $n$. Assume there exists an analytic hypersurface $V \subset Y$ such that $Y-V$ is Stein. Examples of such manifolds are Stein manifolds (where $V$ is empty) and projective algebraic manifolds (where one can take $V$ to be the intersection of $Y$ with a projective hyperplane in some projective space in which $Y$ is embedded).

Fix a smooth hypersurface $Z \subset Y$ such that $Z \not \subset V$. In [V-06] we proved the following generalization of the Ohsawa-Takogoshi Extension Theorem.

TheOREM 4. Suppose given a holomorphic line bundle $H \rightarrow Y$ with a singular Hermitian metric $e^{-\psi}$, and a singular Hermitian metric $e^{-\varphi_{Z}}$ for the line bundle associated to the divisor $Z$, such that the following properties hold.

(i) The restrictions $e^{-\psi} \mid Z$ and $e^{-\varphi_{Z}} \mid Z$ are singular metrics.

(ii) There is a global holomorphic section $T \in H^{0}(Y, Z)$ such that

$$
Z=\{T=0\} \quad \text { and } \quad \sup _{Y}|T|^{2} e^{-\varphi_{Z}}=1 .
$$

(iii) $\sqrt{-1} \partial \bar{\partial} \psi \geq 0$ and there is an integer $\mu>0$ such that $\mu \sqrt{-1} \partial \bar{\partial} \psi \geq \sqrt{-1} \partial \bar{\partial} \varphi_{Z}$. Then for every $s \in H^{0}\left(Z, K_{Z}+H\right)$ such that

$$
\int_{Z}|s|^{2} e^{-\psi}<+\infty \quad \text { and } \quad s \wedge d T \in \mathscr{I}\left(e^{-\left(\varphi_{Z}+\psi\right)} \mid Z\right),
$$

there exists a section $S \in H^{0}\left(Y, K_{Y}+Z+H\right)$ such that

$$
S \mid Z=s \wedge d T \quad \text { and } \quad \int_{Y}|S|^{2} e^{-\left(\varphi_{Z}+\psi\right)} \leq 40 \pi \mu \int_{Z}|s|^{2} e^{-\psi} .
$$


Remark. The list of $L^{2}$ extension theorems is by now rather long. For a large collection of such results and additional references, see [MV-05].

3. Inductive construction of certain sections by extension. In this section we use the method of Paun [P-05] mentioned in the introduction. Fix a holomorphic line bundle $A \rightarrow X$ such that the property $(\mathrm{G})$ in Theorem 1 holds.

Let us fix bases

$$
\left\{\tilde{\sigma}_{j}^{(m, 0, p)} ; 1 \leq j \leq M_{p}\right\}
$$

of $H^{0}\left(X, p\left(K_{X}+Z+E\right)+A\right)$. We let $\sigma_{j}^{(m, 0, p)} \in H^{0}\left(Z, p\left(K_{Z}+E \mid Z\right)+A \mid Z\right)$ be such that

$$
\tilde{\sigma}_{j}^{(m, 0, p)} \mid Z=\sigma_{j}^{(m, 0, p)} \wedge(d T)^{\otimes p} .
$$

We also fix smooth metrics

$$
e^{-\gamma_{Z}} \text { and } e^{-\gamma_{E}} \text { for } Z \rightarrow X, \text { and } E \rightarrow X
$$

respectively. Finally, let us fix bases

$$
s_{1}^{(m)}, \ldots, s_{N_{m}}^{(m)} \text { for } H^{0}\left(X, m\left(K_{Z}+E \mid Z\right)\right), \quad m=1,2, \ldots,
$$

orthonormal with respect to the singular metric $\left(\omega^{-(n-1)} e^{-\gamma_{E}}\right)^{m-1} e^{-\varphi_{E}}$ for $(m-$ 1) $K_{Z}+m E \mid Z$. (Since $e^{-\varphi_{E}}$ is locally integrable, every holomorphic section is integrable with respect to this metric.)

Proposition 5. For each $m=1,2, \ldots$ there exist a constant $C_{m}<+\infty$ and sections

$$
\tilde{\sigma}_{j, \ell}^{(m, k, p)} \in H^{0}\left(X,(k m+p)\left(K_{X}+Z+E\right)+A\right)
$$

where $p=1,2, \ldots, m-1,1 \leq j \leq M_{p}, 1 \leq \ell \leq N_{m}$ and $k=1,2, \ldots$, with the following properties.

(a) $\tilde{\sigma}_{j, \ell}^{(m, k, p)} \mid Z=\left(s_{\ell}^{(m)}\right)^{\otimes k} \otimes \sigma_{j}^{(m, 0, p)} \wedge(d T)^{(k m+p)}$

(b) If $k \geq 1$,

$$
\int_{X} \frac{\sum_{j=1}^{M_{0}}\left|\tilde{\sigma}_{j, \ell}^{(m, k, 0)}\right|^{2} e^{-\left(\gamma_{Z}+\gamma_{E}\right)}}{\sum_{j=1}^{M_{m-1}}\left|\tilde{\sigma}_{j, \ell}^{(m, k-1, m-1)}\right|^{2}} \leq C_{m} .
$$

(c) For $1 \leq p \leq m-1$,

$$
\int_{X} \frac{\sum_{j=1}^{M_{p}}\left|\tilde{\sigma}_{j, \ell}^{(m, k, p)}\right|^{2} e^{-\left(\gamma_{Z}+\gamma_{E}\right)}}{\sum_{j=1}^{M_{p-1}}\left|\tilde{\sigma}_{j, \ell}^{(m, k, p-1)}\right|^{2}} \leq C_{m} .
$$

Proof. (Double induction on $k$ and $p$.) Fix a constant $\widehat{C}_{m}$ such that the

$$
\sup _{X} \frac{\sum_{j=1}^{M_{0}}\left|\tilde{\sigma}_{j}^{(m, 0,0)}\right|^{2} \omega^{n(m-1)} e^{(m-1)\left(\gamma_{Z}+\gamma_{E}\right)}}{\sum_{j=1}^{M_{m-1}}\left|\tilde{\sigma}_{j}^{(m, 0, m-1)}\right|^{2}} \leq \widehat{C}_{m}
$$


and

$$
\sup _{Z} \frac{\sum_{j=1}^{M_{0}}\left|\sigma_{j}^{(m, 0,0)}\right|^{2} \omega^{(n-1)(m-1)} e^{(m-1) \gamma_{E}}}{\sum_{j=1}^{M_{m-1}}\left|\sigma_{j}^{(m, 0, m-1)}\right|^{2}} \leq \widehat{C}_{m},
$$

and for all $0 \leq p \leq m-2$,

$$
\sup _{X} \frac{\sum_{j=1}^{N_{p+1}}\left|\tilde{\sigma}_{j}^{(m, 0, p+1)}\right|^{2} \omega^{-n} e^{-\left(\gamma_{z}+\gamma_{E}\right)}}{\sum_{j=1}^{M_{p}}\left|\tilde{\sigma}_{j}^{(m, 0, p)}\right|^{2}} \leq \widehat{C}_{m},
$$

and

$$
\sup _{Z} \frac{\sum_{j=1}^{N_{p+1}}\left|\sigma_{j}^{(m, 0, p+1)}\right|^{2} \omega^{-(n-1)} e^{-\gamma_{E}}}{\sum_{j=1}^{M_{p}}\left|\sigma_{j}^{(m, 0, p)}\right|^{2}} \leq \widehat{C}_{m} .
$$

$(k=0)$ We set $\tilde{\sigma}_{j, \ell}^{(m, 0, p)}:=\tilde{\sigma}_{j}^{(m, 0, p)}$ and simply observe that

$$
\int_{X} \frac{\sum_{j=1}^{M_{p}}\left|\tilde{\sigma}_{j, \ell}^{(m, 0, p)}\right|^{2} e^{-\left(\gamma_{Z}+\gamma_{E}\right)}}{\sum_{j=1}^{M_{p-1}}\left|\tilde{\sigma}_{j, \ell}^{(m, 0, p-1)}\right|^{2}} \leq \widehat{C}_{m} \int_{X} \omega^{n} .
$$

$(k \geq 1)$ Assume the result has been proved for $k-1$.

$\underline{((p=0))}$ : Consider the sections $\left(s_{\ell}^{(m)}\right)^{\otimes k} \otimes \sigma_{j}^{(m, 0,0)}$, and define the semi-positively curved metric

$$
\psi_{k, \ell, 0}:=\log \sum_{j=1}^{M_{m-1}}\left|\tilde{\sigma}_{j, \ell}^{(m, k-1, m-1)}\right|^{2}
$$

for the line bundle $(m k-1)\left(K_{X}+Z+E\right)+A$. Observe that locally on $Z$,

$$
\begin{aligned}
\left|\left(s_{\ell}^{(m)} \wedge d T^{m}\right)^{k} \otimes \sigma_{j}^{(m, 0,0)}\right|^{2} e^{-\left(\varphi_{Z}+\psi_{k, \ell, 0}+\varphi_{E}\right)} & =\left|s_{\ell}^{(m)} \wedge d T^{m}\right|^{2} \frac{\left|\sigma_{j}^{(m, 0,0)}\right|^{2} e^{-\left(\varphi_{Z}+\varphi_{E}\right)}}{\sum_{j=1}^{M_{m}-1}\left|\sigma_{j}^{(m, 0, m-1)}\right|^{2}} \\
& \lesssim\left|s_{\ell}^{(m)}\right|^{2} e^{-\left(\varphi_{Z}+\varphi_{E}\right)}
\end{aligned}
$$

Moreover, we have

$$
\sqrt{-1} \partial \bar{\partial}\left(\psi_{k, \ell, 0}+\varphi_{E}\right) \geq 0 \quad \text { and } \quad \mu \sqrt{-1} \partial \bar{\partial}\left(\psi_{k, \ell, 0}+\varphi_{E}\right) \geq \sqrt{-1} \partial \bar{\partial} \varphi_{Z} .
$$

Finally,

$$
\begin{aligned}
& \int_{Z}\left|\left(s_{\ell}^{(m)}\right)^{k} \otimes \sigma_{j}^{(m, 0,0)}\right|^{2} e^{-\left(\psi_{k, \ell, 0}+\varphi_{E}\right)} \\
= & \int_{Z}\left|s_{\ell}^{(m)}\right|^{2} \frac{\left|\sigma_{j}^{(m, 0,0)}\right|^{2} e^{(m-1) \gamma_{E}} e^{-\left((m-1) \gamma_{E}+\varphi_{E}\right)}}{\sum_{j=1}^{M_{m-1}}\left|\sigma_{j}^{(m, 0, m-1)}\right|^{2}}<+\infty .
\end{aligned}
$$

We may thus apply Theorem 4 to obtain sections

$$
\tilde{\sigma}_{j, \ell}^{(m, k, 0)} \in H^{0}\left(X, m k\left(K_{X}+Z+E\right)+A\right), \quad 1 \leq j \leq M_{0}, 1 \leq \ell \leq N_{m},
$$


such that

$$
\tilde{\sigma}_{j, \ell}^{(m, k, 0)} \mid Z=\left(s_{\ell}^{(m)}\right)^{\otimes k} \otimes \sigma_{j, \ell}^{(m, 0,0)} \wedge(d T)^{\otimes k m}, \quad 1 \leq j \leq M_{0}, 1 \leq \ell \leq N_{m},
$$

and

$$
\int_{X}\left|\tilde{\sigma}_{j, \ell}^{(m, k, 0)}\right|^{2} e^{-\left(\psi_{k, \ell, 0}+\varphi_{Z}+\varphi_{E}\right)} \leq 40 \pi \mu \int_{Z}\left|s_{\ell}^{(m)}\right|^{2} \frac{\left|\sigma_{j}^{(0)}\right|^{2} e^{-\left(\varphi_{E}+\varphi_{B}\right)}}{\sum_{j=1}^{N_{m-1}}\left|\sigma_{j}^{(m-1)}\right|^{2}} .
$$

Summing over $j$, we obtain

$$
\begin{aligned}
& \int_{X} \frac{\sum_{j=1}^{M_{0}}\left|\tilde{\sigma}_{j, \ell}^{(m, k, 0)}\right|^{2} e^{-\left(\gamma_{Z}+\gamma_{E}\right)}}{\sum_{j=1}^{M_{m-1}}\left|\tilde{\sigma}_{j, \ell}^{(m, k-1, m-1)}\right|^{2}} \\
\leq & \sup _{X} e^{\varphi_{Z}+\varphi_{E}-\gamma_{Z}-\gamma_{E}} \int_{X} \frac{\sum_{j=1}^{M_{0}}\left|\tilde{\sigma}_{j, \ell}^{(m, k, 0)}\right|^{2} e^{-\left(\varphi_{Z}+\varphi_{E}\right)}}{\sum_{j=1}^{M_{m-1}}\left|\tilde{\sigma}_{j, \ell}^{(m, k-1, m-1)}\right|^{2}} \\
\leq & 40 \pi \sup _{X} e^{\varphi_{Z}+\varphi_{E}-\gamma_{Z}-\gamma_{E}} \int_{Z}\left|s_{\ell}^{(m)}\right|^{2} \frac{\sum_{j=1}^{M_{0}}\left|\sigma_{j}^{(m, 0,0)}\right|^{2} e^{-\varphi_{E}}}{\sum_{j=1}^{M_{m}-1}\left|\sigma_{j}^{(m, 0, m-1)}\right|^{2}} e^{-\kappa} \\
\leq & 40 \pi \widehat{C}_{m} \sup _{X} e^{\varphi_{Z}+\varphi_{E}-\gamma_{Z}-\gamma_{E}} \int_{Z}\left|s_{\ell}^{(m)}\right|^{2} \omega^{-(n-1)(m-1)} e^{-\left((m-1) \gamma_{E}+\varphi_{E}\right)} \\
= & 40 \pi \widehat{C}_{m} \sup _{X} e^{\varphi_{Z}+\varphi_{E}-\gamma_{Z}-\gamma_{E}} .
\end{aligned}
$$

$((1 \leq p \leq m-1)):$ Assume that we have obtained the sections $\tilde{\sigma}_{j, \ell}^{(m, k, p-1)}, 1 \leq j \leq$ $M_{p-1}, 1 \leq \ell \leq N_{m}$. Consider the non-negatively curved singular metric

$$
\psi_{k, \ell, p}:=\log \sum_{j=1}^{M_{p-1}}\left|\tilde{\sigma}_{j, \ell}^{(m, k, p-1)}\right|^{2}
$$

for $(k m+p-1)\left(K_{X}+Z+E\right)+A$. We have

$$
\left|\left(s_{\ell}^{(m)}\right)^{k} \otimes \sigma_{j}^{(m, 0, p)}\right|^{2} e^{-\left(\varphi_{Z}+\psi_{k, \ell, p}+\varphi_{E}\right)}=\frac{\left|\sigma_{j}^{(m, 0, p)}\right|^{2} e^{-\left(\varphi_{Z}+\varphi_{E}\right)}}{\sum_{j=1}^{M_{p-1}}\left|\sigma_{j}^{(m, 0, p-1)}\right|^{2}} \lesssim e^{-\left(\varphi_{Z}+\varphi_{E}\right)},
$$

which is locally integrable on $Z$ by the hypothesis $(\mathrm{T})$. Next,

$$
\begin{aligned}
\int_{Z}\left|\left(s_{\ell}^{(m)}\right)^{k} \otimes \sigma_{j}^{(m, 0, p)}\right|^{2} e^{-\left(\psi_{k, \ell, p}+\varphi_{E}\right)} & =\int_{Z} \frac{\left|\sigma_{j}^{(m, 0, p)}\right|^{2} e^{-\varphi_{E}}}{\sum_{j=1}^{M_{p-1}}\left|\sigma_{j}^{(m, 0, p-1)}\right|^{2}} \\
& \leq C^{\star} \int_{Z} e^{\gamma_{Z}} \frac{\left|\sigma_{j}^{(m, 0, p)}\right|^{2} e^{-\left(\varphi_{Z}+\varphi_{E}\right)}}{\sum_{j=1}^{M_{p-1}}\left|\sigma_{j}^{(m, 0, p-1)}\right|^{2}}<+\infty,
\end{aligned}
$$

where

$$
C^{\star}:=\sup _{Z} e^{\varphi_{Z}-\gamma_{Z}}
$$

Moreover,

$$
\sqrt{-1} \partial \bar{\partial}\left(\psi_{k, \ell, p}+\varphi_{E}\right) \geq 0 \quad \text { and } \quad \sqrt{-1} \partial \bar{\partial}\left(\psi_{k, \ell, p}+\varphi_{E}\right) \geq \sqrt{-1} \partial \bar{\partial} \varphi_{Z}
$$


By Theorem 4 there exist sections

$$
\tilde{\sigma}_{j, \ell}^{(m, k, p)} \in H^{0}\left(X,(m k+p)\left(K_{X}+Z+E\right)+A\right), \quad 1 \leq j \leq M_{0}
$$

such that

$$
\tilde{\sigma}_{j, \ell}^{(m, k, p)} \mid Z=\left(s_{\ell}^{(m)}\right)^{\otimes k} \otimes \sigma_{j, \ell}^{(m, 0, p)} \wedge(d T)^{\otimes k m+p}, \quad 1 \leq j \leq M_{p},
$$

and

$$
\int_{X}\left|\tilde{\sigma}_{j, \ell}^{(m, k, p)}\right|^{2} e^{-\left(\psi_{k, \ell, p}+\varphi_{Z}+\varphi_{E}\right)} \leq 40 \pi \mu \int_{Z} \frac{\left|\sigma_{j}^{(m, 0, p)}\right|^{2} e^{-\varphi_{E}}}{\sum_{j=1}^{M_{p-1}}\left|\sigma_{j}^{(m, 0, p-1)}\right|^{2}} .
$$

Summing over $j$, we obtain

$$
\int_{X} \frac{\sum_{j=1}^{M_{p}}\left|\tilde{\sigma}_{j, \ell}^{(m, k, p)}\right|^{2} e^{-\left(\gamma_{Z}+\gamma_{E}\right)}}{\sum_{j=1}^{M_{p-1}}\left|\tilde{\sigma}_{j, \ell}^{(m, k, p-1)}\right|^{2}} \leq 40 \pi \mu \sup _{X} e^{\varphi_{Z}+\varphi_{E}-\gamma_{Z}-\gamma_{E}} \widehat{C}_{m} \int_{Z} e^{-\varphi_{E}} \omega^{n-1} .
$$

Letting

$$
:=40 \pi \mu \widehat{C}_{m} \max \left(\int_{X} \omega^{n}, \sup _{X} e^{\varphi_{Z}+\varphi_{E}+\varphi_{B}-\gamma_{Z}-\gamma_{E}}, \sup _{X} e^{\varphi_{Z}+\varphi_{E}-\gamma_{Z}-\gamma_{E}} \int_{Z} e^{-\varphi_{E}} \omega^{n-1}\right)
$$

completes the proof.

4. Construction of the metric. This part of the proof follows the ideas of Siu set forth in [S-02].

4.1. A metric associated to $\mathbf{m}\left(\mathbf{K}_{\mathbf{X}}+\mathbf{Z}+\mathbf{E}\right)$. Fix a smooth metric $e^{-\psi}$ for $A \rightarrow X$. Consider the functions

$$
\lambda_{\ell, N}^{(m)}:=\log \sum_{j=1}^{M_{p}}\left|\tilde{\sigma}_{j, \ell}^{(m, k, p)}\right|^{2} \omega^{-n(m k+p)} e^{-\left(k m\left(\gamma_{Z}+\gamma_{E}\right)+\psi\right)},
$$

where $N=m k+p$. Set

$$
\lambda_{N}^{(m)}:=\log \sum_{\ell=1}^{N_{m}} e^{\lambda_{\ell, N}^{(m)}}
$$

Lemma 6. For any non-empty open subset $V \subset X$ and any smooth function $f: \bar{V} \rightarrow \mathbb{R}_{+}$,

$$
\frac{1}{\int_{V} f \omega^{n}} \int_{V}\left(\lambda_{N}^{(m)}-\lambda_{N-1}^{(m)}\right) f \omega^{n} \leq \log \left(\frac{N_{m} C_{m} \sup _{V} f}{\int_{V} f \omega^{n}}\right) .
$$

Proof. Observe that by Proposition 5, there exists a constant $C_{m}$ such that for any open subset $V \subset X$,

$$
\int_{V}\left(e^{\lambda_{\ell, N}^{(m)}-\lambda_{\ell, N-1}^{(m)}}\right) f \omega^{n} \leq C_{m} \sup _{V} f
$$


and thus

$$
\int_{V}\left(e^{\lambda_{N}^{(m)}-\lambda_{N-1}^{(m)}}\right) f \omega^{n}=\sum_{\ell=1}^{N_{m}} \int_{V}\left(e^{\lambda_{\ell, N}^{(m)}-\lambda_{\ell, N-1}^{(m)}}\right) f \omega^{n} \leq N_{m} C_{m} \sup _{V} f .
$$

An application of (the concave version of) Jensen's inequality to the concave function log then gives

$$
\frac{1}{\int_{V} f \omega^{n}} \int_{V}\left(\lambda_{N}^{(m)}-\lambda_{N-1}^{(m)}\right) f \omega^{n} \leq \log \left(\frac{N_{m} C_{m} \sup _{V} f}{\int_{V} f \omega^{n}}\right) .
$$

The proof is complete.

Consider the function

$$
\Lambda_{k}^{(m)}=\frac{1}{k} \lambda_{m k}^{(m)} .
$$

Note that $\Lambda_{k}^{(m)}$ is locally the sum of a plurisubharmonic function and a smooth function. By applying Lemma 6 and using the telescoping property, we see that for any open set $V \subset X$ and any smooth function $f: \bar{V} \rightarrow \mathbb{R}_{+}$,

$$
\frac{1}{\int_{V} f \omega^{n}} \int_{V} \Lambda_{k}^{(m)} f \omega^{n} \leq m \log \left(\frac{N_{m} C_{m} \sup _{V} f}{\int_{V} f \omega^{n}}\right) .
$$

Proposition 7. There exists a constant $C_{o}^{(m)}$ such that

$$
\Lambda_{k}^{(m)}(x) \leq C_{o}^{(m)}, \quad x \in X .
$$

Proof. Let us cover $X$ by coordinate charts $V_{1}, \ldots, V_{N}$ such that for each $j$ there is a biholomorphic map $F_{j}$ from $V_{j}$ to the ball $B(0,2)$ of radius 2 centered at the origin in $\mathbb{C}^{n}$, and such that if $U_{j}=F_{j}^{-1}(B(0,1))$, then $U_{1}, \ldots, U_{N}$ is also an open cover. Let $W_{j}=V_{j} \backslash F_{j}^{-1}(B(0,3 / 2))$.

Now, on each $V_{j}, \Lambda_{k}^{(m)}$ is the sum of a plurisubharmonic function and a smooth function. Say $\Lambda_{k}^{(m)}=h+g$ on $V_{j}$, where $h$ is plurisubharmonic and $g$ is smooth. Then for constant $A_{j}$ we have

$$
\begin{aligned}
\sup _{U_{j}} \Lambda_{k}^{(m)} & \leq \sup _{U_{j}} g+\sup _{U_{j}} h \\
& \leq \sup _{U_{j}} g+A_{j} \int_{W_{j}} h \cdot F_{j *} d V \\
& \leq \sup _{U_{j}} g-A_{j} \int_{W_{j}} g \cdot F_{j *} d V+A_{j} \int_{W_{j}} \Lambda_{k}^{(m)} \cdot F_{j *} d V
\end{aligned}
$$

Let

$$
C_{j}^{(m)}:=\sup _{U_{j}} g-A_{j} \int_{W_{j}} g \cdot F_{j *} d V
$$

and define the smooth function $f_{j}$ by

$$
f_{j} \omega^{n}=F_{j *} d V .
$$


Then by (1) applied with $V=W_{j}$ and $f=f_{j}$, we have

$$
\sup _{U_{j}} \Lambda_{k}^{(m)} \leq C_{j}^{(m)}+m A_{j} \log \left(\frac{N_{m} C_{m} \sup _{W_{j}} f_{j}}{\int_{W_{j}} f_{j} \omega^{n}}\right) \int_{W_{j}} f_{j} \omega^{n} .
$$

Letting

$$
C_{o}^{(m)}:=\max _{1 \leq j \leq N}\left\{C_{j}^{(m)}+m A_{j} \log \left(\frac{N_{m} C_{m} \sup _{W_{j}} f_{j}}{\int_{W_{j}} f_{j} \omega^{n}}\right) \int_{W_{j}} f_{j} \omega^{n}\right\}
$$

completes the proof.

Since the upper regularization of the lim sup of a uniformly bounded sequence of plurisubharmonic functions is plurisubharmonic (see, e.g., [H-90, Theorem 1.6.2]), we essentially have the following corollary.

COROllary 8. The function

$$
\Lambda^{(m)}(x):=\limsup _{y \rightarrow x} \limsup _{k \rightarrow \infty} \Lambda_{k}^{(m)}(y)
$$

is locally the sum of a plurisubharmonic function and a smooth function.

Proof. One need only observe that the function $\Lambda_{k}$ is obtained from a singular metric on the line bundle $m\left(K_{X}+Z+E\right)$ (this singular metric $e^{-\kappa_{k}^{(m)}}$ will be described shortly) by multiplying by a fixed smooth metric of the dual line bundle. $\square$

Consider the singular Hermitian metric $e^{-\kappa^{(m)}}$ for $m\left(K_{X}+Z+E\right)$ defined by

$$
e^{-\kappa^{(m)}}=e^{-\Lambda^{(m)}} \omega^{-n m} e^{-m\left(\gamma_{Z}+\gamma_{E}\right)} .
$$

This singular metric is given by the formula

$$
e^{-\kappa^{(m)}(x)}=\exp \left(-\limsup _{y \rightarrow x} \limsup _{k \rightarrow \infty} \kappa_{k}^{(m)}(y)\right)
$$

where

$$
e^{-\kappa_{k}^{(m)}}=e^{-\Lambda_{k}^{(m)}} \omega^{-n m} e^{-m\left(\gamma_{Z}+\gamma_{E}\right)} .
$$

The curvature of $e^{-\kappa_{k}^{(m)}}$ is thus

$$
\begin{aligned}
\sqrt{-1} \partial \bar{\partial} \kappa_{k}^{(m)} & =\frac{\sqrt{-1}}{k} \partial \bar{\partial} \log \sum_{\ell=1}^{N_{m}} \sum_{j=1}^{N_{0}}\left|\tilde{\sigma}_{j, \ell}^{(m, k, 0)}\right|^{2}-\frac{1}{k} \sqrt{-1} \partial \bar{\partial} \psi \\
& \geq-\frac{1}{k} \sqrt{-1} \partial \bar{\partial} \psi .
\end{aligned}
$$

We claim next that the curvature of $e^{-\kappa}$ is non-negative. To see this, it suffices to work locally. Then we have that the functions

$$
\kappa_{k}^{(m)}+\frac{1}{k} \psi
$$

are plurisubharmonic. But

$$
\limsup _{y \rightarrow x} \limsup _{k \rightarrow \infty} \kappa_{k}^{(m)}+\frac{1}{k} \psi=\limsup _{y \rightarrow x} \limsup _{k \rightarrow \infty} \kappa_{k}^{(m)}=\kappa^{(m)} .
$$

It follows that $\kappa^{(m)}$ is plurisubharmonic, as desired. 
4.2. The metric for $\mathbf{K}_{\mathbf{X}}+\mathbf{Z}+\mathbf{E}$; Proof of Theorem 1. Let $\varepsilon_{m}$ be constants, chosen so $\varepsilon_{m} \searrow 0$ sufficiently rapidly that the sum

$$
e^{\kappa}:=\sum_{m=1}^{\infty} \varepsilon_{m} e^{\frac{1}{m} \kappa^{(m)}}=\sum_{m=1}^{\infty} \exp \left(\frac{1}{m} \kappa^{(m)}+\log \varepsilon_{m}\right),
$$

converges everywhere on $X$ (to a metric for $-\left(K_{X}+Z+E\right)$ ). It is possible to find such constants since, by Proposition 7 , each $\kappa^{(m)}$ is locally uniformly bounded from above. (The lower bound $e^{\kappa^{(m)}} \geq 0$ is trivial.) Moreover, by elementary properties of plurisubharmonic functions, $\kappa$ is plurisubharmonic. Indeed, for any $r \in \mathbb{N}$, the function

$$
\psi_{r}:=\log \sum_{m=1}^{r} \exp \left(\frac{1}{m} \kappa^{(m)}+\log \varepsilon_{m}\right)
$$

is plurisubharmonic, and $\psi_{r} \nearrow \kappa$. It follows that $\kappa=\sup _{r} \psi_{r}$ is plurisubharmonic. (Again, see [H-90, Theorem 1.6.2].) Thus $e^{-\kappa}$ is a singular Hermitian metric for $K_{X}+$ $Z+E$ with non-negative curvature current.

Observe that, after identifying $K_{Z}$ with $\left(K_{X}+Z\right) \mid Z$ by dividing by $d T$,

$$
\left.\kappa_{k}^{(m)}\left|Z=\log \left(\sum_{\ell=1}^{N_{m}}\left|s_{\ell}^{(m)}\right|^{2}\right)+\frac{1}{k} \log \sum_{j=1}^{M_{0}}\right| \sigma_{j}^{(m, 0,0)}\right|^{2} .
$$

Thus we obtain $e^{-\kappa^{(m)}} \mid Z=\left(\sum_{\ell=1}^{N_{m}}\left|s_{\ell}^{(m)}\right|^{2}\right)^{-1}$. It follows that

$$
e^{-\kappa} \mid Z=\frac{1}{\sum_{m=1}^{\infty} \varepsilon_{m}\left(\sum_{\ell=1}^{N_{m}}\left|s_{\ell}^{(m)}\right|^{2}\right)^{2 / m}} .
$$

In view of the short discussion following the proof of Proposition 3, the metric $e^{-\kappa}$ satisfies the conclusions of Theorem 1. The proof of Theorem 1 is thus complete.

Acknowledgement. I am indebted to Lawrence Ein and Mihnea Popa. It is to a discussion with them that the present paper owes its existence.

\section{REFERENCES}

[C-06] Claudon, B., Invariance of multiples of the twisted canonical bundle, preprint 2006.

[H-90] Hörmander, L., An introduction to complex analysis in several variables, Third edition. North-Holland Mathematical Library, 7. North-Holland Publishing Co., Amsterdam, 1990.

[MV-05] McNeal, J.; VArolin, D., Analytic inversion of adjunction: $L^{2}$ extension theorems with gain, preprint 2005. To appear in Ann. Inst. Fourier.

[P-05] PAun, M., Siu's invariance of plurigenera: a one-tower proof, preprint 2005.

[S-98] SIU, Y.-T., Invariance of plurigenera, Invent. Math., 134:3 (1998), pp. 661-673.

[S-02] SiU, Y.-T., Extension of twisted pluricanonical sections with plurisubharmonic weight and invariance of semipositively twisted plurigenera for manifolds not necessarily of general type, Complex geometry. Collection of papers dedicated to Hans Grauert. Springer-Verlag, Berlin, 2002, pp. 223-277.

[Ta-05] TAKAYAma, S, Pluricanonical systems on algebraic varieties of general type, to appear in Compositio Math.

[Ts-02] TsujI, H., Deformation invariance of plurigenera, Nagoya Math. J., 166 (2002), pp. 117134.

[V-06] VArolin, D., A Takayama-type Extension Theorem, preprint 2006. 
D. VAROLIN 